\title{
The metabolic profile of a rat model of chronic kidney disease
}

\author{
Yohei Tanada $^{1}{ }^{\text {, Junji Okuda }}{ }^{1}$, Takao Kato ${ }^{\text {Corresp., }}{ }^{1}$, Eri Minamino-Muta ${ }^{1}$, Ichijiro Murata ${ }^{2}$, Tomoyoshi Soga $^{3}$ \\ , Tetsuo Shioi ${ }^{1}$, Takeshi Kimura ${ }^{1}$ \\ ${ }^{1}$ Department of Cardiovascular Medicine, Graduate School of Medicine, Kyoto University, Kyoto, Japan \\ 2 \\ 3 Institute for Advanced Biosciences, Keio University, Tsuruoka, Japan \\ Corresponding Author: Takao Kato \\ Email address: tkato75@kuhp.kyoto-u.ac.jp
}

Background: The kidney is always subjected to high metabolic demand. The aim of this study was to characterize metabolic profiles of a rat model of chronic kidney disease (CKD) with cardiorenal syndrome (CRS) induced by prolonged hypertension.

Methods: We used inbred male Dahl salt-sensitive (DS) rats fed an $8 \% \mathrm{NaCl}$ diet from 6 weeks of age (high-salt; HS group) or a $0.3 \% \mathrm{NaCl}$ diet as controls (low-salt; LS group). We analyzed function, pathology, metabolome, and the gene expression related to energy metabolism of the kidney.

Results: DS rats with a high-salt diet showed hypertension at 11 weeks of age and elevated serum levels of creatinine and blood urea nitrogen with heart failure at 21 weeks of age. The fibrotic area in the kidneysincreased at 21 weeks of age. In addition, gene expression related to mitochondrial function was largely decreased. The levels of citrate and isocitrate increased and the gene expression of alphaketoglutaratedehydrogenase and succinyl-CoA synthetase decreased; these are enzymes that metabolize citrate and isocitrate, respectively. In addition, the levels of succinate and acetyl Co-A, both of which are metabolites of the tricarboxylic acid (TCA) cycle, decreased.

Conclusions: DS rats fed a high-salt diet were deemed a suitable model of CKD with CRS. Gene expression and metabolites related to energy metabolism and mitochondria in the kidney significantly changed in DS rats with hypertension in accordance with the progression of renal injury. 


\section{The metabolic profile of a rat model of chronic kidney disease}

2 Yohei Tanada ${ }^{1 \#,}$ Junji Okuda ${ }^{1 \#}$, Takao Kato ${ }^{1 *}$, Eri Minamino-Muta ${ }^{1}$, Ichijiro Murata ${ }^{2}$, Tomoyoshi

3 Soga $^{3}$, Tetsuo Shioi ${ }^{1}$, Takeshi Kimura ${ }^{1}$

4 1. Department of Cardiovascular Medicine, Graduate School of Medicine, Kyoto University, 54

5 Kawahara-cho, Sakyou-ku, Kyoto City, Kyoto, Japan

6 2. Department of Nephrology, Gifu University Graduate School of Medicine, 1-1 Yanagido, Gifu

7 City, Gifu, Japan

8 3. Institute for Advanced Biosciences, Keio University, Kakuganji Mizukami 246-2, Tsuruoka

9 City, Yamagata, Japan

10 \# These authors contributed equally to this work.

11 Corresponding author: Takao Kato

12 Department of Cardiovascular Medicine, Graduate School of Medicine, Kyoto University, 54

13 Shogoin Kawahara-cho, Sakyo-ku, Kyoto, 606-8507 Japan

14 Tel: +81-75-751-3190; Fax: +81-75-751-3203

15 Email: tkato75@kuhp.kyoto-u.ac.jp (TK) 


\section{Abstract}

17 Background: The kidney is always subjected to high metabolic demand. The aim of this study was to characterize metabolic profiles of a rat model of chronic kidney disease (CKD) with cardiorenal syndrome (CRS) induced by prolonged hypertension.

Methods: We used inbred male Dahl salt-sensitive (DS) rats fed an $8 \% \mathrm{NaCl}$ diet from 6 weeks

21 of age (high-salt; HS group) or a $0.3 \% \mathrm{NaCl}$ diet as controls (low-salt; LS group). We analyzed function, pathology, metabolome, and the gene expression related to energy metabolism of the

23 kidney.

Results: DS rats with a high-salt diet showed hypertension at 11 weeks of age and elevated serum levels of creatinine and blood urea nitrogen with heart failure at 21 weeks of age. The fibrotic area in the kidneys increased at 21 weeks of age. In addition, gene expression related to mitochondrial function was largely decreased. The levels of citrate and isocitrate increased and the gene expression of alpha-ketoglutarate dehydrogenase and succinyl-CoA synthetase decreased; these are enzymes that metabolize citrate and isocitrate, respectively. In addition, the levels of succinate and acetyl Co-A, both of which are metabolites of the tricarboxylic acid

31 (TCA) cycle, decreased. 
32 Conclusions: DS rats fed a high-salt diet were deemed a suitable model of CKD with CRS. Gene

33 expression and metabolites related to energy metabolism and mitochondria in the kidney

34 significantly changed in DS rats with hypertension in accordance with the progression of renal

35 injury. 


\section{Introduction}

37 Chronic kidney disease (CKD) is a worldwide public health problem that affects millions of

38 people worldwide. There has been an increase in the prevalence of CKD in developed and

39 developing countries. The Global Burden of Disease Study revealed that CKD was ranked 27th

40 on the list of causes of total number of global deaths in 1990, but rose to 18th in 2010 (Lozano et

41 al. 2012). Patients with CKD have a ten-fold increase in incidence of cardiovascular death due to

42 common underlying causes of cardiovascular diseases (Hillege et al 2000; Lozano et al. 2012;

43 Smith et al 2006), and impaired renal function is an independent risk factor for morbidity and

44 mortality in patients with heart failure (HF) (Hillege et al. 2000; Smith et al. 2006). Therefore,

45 the term cardiorenal syndrome (CRS) has been introduced in recent years to characterize this

46 association.

Many risk factors for kidney disease progression have been identified, and poorly controlled

hypertension is a major predictor (Eirin \& Lerman 2013; Nourbakhsh \& Singh 2014; Rubattu et

al. 2013). Studies have highlighted several activated deleterious pathways in the hypertensive

51 kidney. Inflammation, oxidative stress, the renin-angiotension-aldosterone system (RAAS), and

fibrosis are proposed underling mechanisms eliciting functional decline (Bidani \& Griffin 2004; 
53 Eirin \& Lerman 2013). Moreover, in CRS, the same deleterious pathways develop vicious cycle,

54 which CKD and HF exacerbate each other (Rubattu et al. 2013). However, the precise molecular

55 mechanisms responsible for renal injury in CRS have not been fully elucidated. The kidney is a

56 highly energetic organ that is richly perfused. Renal blood flow (RBF) normally accounts for

57 approximately $25 \%$ of cardiac output. The renal oxygen consumption per gram of tissue is the

58 second-highest compared to that of other organs, with cardiac oxygen consumption being the

59 highest (Nourbakhsh \& Singh 2014). This is largely driven by the high RBF since the renal

60 oxygen extraction is low mitochondrial homeostasis is critical for the maintenance of normal

61 kidney function, including the production of cellular energy and the regulation of reactive

62 oxygen species. Thus, metabolic protection against acute kidney injury has been intensively

63 investigated, such as activators of mitochondrial biogenesis and ROS modulators (Ishimoto \&

64 Inagi 2015). However, there are few studies that have explored the influence of metabolic

65 changes of CKD in CRS.

67 The Dahl salt-sensitive (DS) rat is a useful model system for understanding salt-induced hypertension, since these rats exhibit marked hypertension when they are fed a high-salt diet

69 (Inoko et al. 1994; Kato et al. 2010). Specifically, when the rats are fed a high-salt diet, they 
70 develop hypertension and subsequently develop HF at approximately 18 weeks of age (Kato et

71 al. 2010). The DS rats fed a high-salt diet also show an increase of serum creatinine and urinary

72 protein expression related to kidney injury at approximately 10 weeks, and can be used as a

73 model of CKD (Hisaki et al. 2005; Nishiyama et al. 2004). The purpose of the present study was-

74 to characterize the change of renal metabolic profiles using DS rats with hypertension at 21

75 weeks of age, which is the end stage of CKD and HF. This was done to gain insight into

76 potential therapeutic targets for CKD in CRS. 


\section{Methods}

\section{Animals}

Animal care and experiments were approved by the Institutional Animal Care and Use Committee of Kyoto University (MedKyo 14183) and conducted following the Guide for Care and Use of Laboratory Animals published by the United States National Institutes of Health. Inbred male DS rats (Japan SLC, Hamamatsu, Shizuoka, Japan) were fed a 0.3\% NaCl (low-salt; LS) diet until the age of 6 weeks, after which they were fed an $8 \% \mathrm{NaCl}$ diet (high-salt; $\mathrm{HS}$ ) (Inoko et al. 1994; Kato et al. 2010; Kawamoto et al. 2015). The rats in the current study were the same animals used in our previous report (Tanada et al. 2015). Therefore, the characterization of hemodynamics in these rats has already been reported.

\section{Protocols}

DS rats fed only an LS diet (LS group) were used as controls, and DS rats fed an HS diet (HS group) were used as a model of renal failure. There were four groups of rats categorized by the amount of salt in the diet (LS or HS) and week of sacrifice (11 weeks or 21 weeks): the 11-weekold LS group ( $n=8), 11$-week-old HS group (n=8), 21-week-old LS group $(n=12)$, and 21-weekold HS group $(n=11)$. Serial measurements of heart rate and blood pressure were performed 
95 every 2 weeks from the age of 11 weeks until sacrifice at 21 weeks.

96

97 Blood and tissue sampling

98 The rats were anesthetized with inhaled diethyl ether (Wako Pure Chemical Industries, Osaka,

99 Japan) and sacrificed by decapitation without fasting. Blood samples were collected via the right

100 atrium in tubes and centrifuged at $1500 \mathrm{rpm}$ for $10 \mathrm{~min}$, and blood serum was taken from the

101 supernatant. Measurement of serum creatinine and blood urea nitrogen (BUN) was entrusted to

102 SRL Inc. (Kyoto, Japan). Both kidneys were rapidly removed, snap frozen in liquid nitrogen, and

103 stored at $-80^{\circ} \mathrm{C}$ or fixed with $4 \%$ paraformaldehyde (PFA).

104

105 Cardiac echocardiography

106 Transthoracic echocardiography was performed as previously reported (Kato et al. 2010). Rats

107 were anesthetized briefly with inhaled diethyl ether (Wako Pure Chemical Industries, Osaka,

108 Japan), and transthoracic echocardiography was performed using a Sonos-5500 echocardiograph

109 (Agilent Technologies, Santa Clara, CA, USA) with a 15-MHz linear transducer. Intraventricular

110 septal thickness (IVSd), left ventricular dimension in the diastolic phase (LVDd), and left

111 ventricular dimension in the systolic phase (LVDs) were measured with M-mode

112 echocardiography, and fractional shortening (FS) was calculated. 


\section{Quantitative reverse transcription-polymerase chain reaction}

115 Total RNA was isolated from the kidneys in each group by the acid guanidinium thiocyanate-

116 phenol-chloroform method. Quantitative reverse transcription-polymerase chain reaction (RT-

117 PCR) was performed as in previous reports (Inuzuka et al. 2009; Niizuma et al. 2012). The

118 oligonucleotide primers are listed in Supplementary Table 1. The mRNA level of each gene was

119 standardized using the expression level of the $18 \mathrm{~S}$ ribosomal RNA as a control.

\section{Fibrosis of the kidneys}

122 The kidneys were fixed in 4\% PFA, embedded in paraffin, and sectioned for histological

123 evaluation. The fibrotic area was quantified in tissue sections with Sirius Red staining, as

124 previously described (Okuda et al. 2013).

\section{Metabolomic analysis of the kidneys}

127 The kidneys were subjected to metabolomic analysis as described previously (Kato et al. 2010)

128 using capillary electrophoresis time-of-flight mass spectrometry (CE-TOFMS) (Soga et al. 2003). Briefly, frozen kidney tissues were immediately plunged into methanol $(0.5 \mathrm{ml})$ containing internal standards (300 $\mu \mathrm{M}$ each of methionine sulfate for cations and MES for anions) and 
131 homogenized for 3 min to inactivate enzymes. $200 \mu 1$ of deionized water and $500 \mu 1$ of

132 chloroform were added, and the mixture was thoroughly mixed. The solution was centrifuged at

$13315,000 \mathrm{rpm}$ for $15 \mathrm{~min}$ at $4{ }^{\circ} \mathrm{C}$ and the $600 \mu \mathrm{l}$ upper aqueous layer was filtered centrifugally

134 through a Milipore-5kDa cutoff filter to remove proteins. All CE-TOFMS was performed using

135 an Agilent CE capillary electrophoresis system (Agilent Technologies, Santa Clara, CA). We

136 measured the levels of metabolites in glycolysis and the tricarboxylic acid (TCA) cycle, and

137 amino acids and their derivatives.

138

\section{Western blotting}

140 Western blotting of kidney tissues was performed as described previously (Tanada et al. 2015).

141 The primary antibodies used for Western blotting were as follows: NDUFA9 (1:1,000, Santa

142 Cruz, Dallas, TX, USA) and GAPDH (1:1,000, Cell Signaling, Danvers, MA, USA).

\section{Statistical analysis}

145 Values are expressed as means \pm the standard error of the mean (SEM). ANOVA was used for

146 comparisons multiple groups. In all tests, a value of $\mathrm{p}<0.05$ was considered statistically

147 significant. 
148

149

150

151

152

153

154

155

156

157

158

159

160

161

162

163

164

\section{Results}

Dahl salt-sensitive rats fed a high-salt diet show a high heart rate, systolic blood pressure, and cardiac dysfunction.

As we previously reported (Tanada et al. 2015), DS rats fed an HS diet (HS group) developed hypertension, showed cardiac dysfunction, and died from heart failure. DS rats fed an LS diet

(LS group) did not develop hypertension, and were used as controls. The heart rate was significantly higher in the HS group compared to the LS group from 11 weeks of age to 19 weeks of age (Supplementary Figure 1A). Conversely, systolic blood pressure was significantly higher in the HS group compared to LS group from 11 weeks of age to 21 weeks of age (Supplementary Figure 1A). At 21 weeks of age, intraventricular septal thickness (IVSd) increased in the HS group on echocardiographic examination. In addition, left ventricular dimension in the diastolic phase (LVDd) increased, and fractional shortening (FS) decreased in the HS group compared to that of the LS group (Supplementary Figure 1B).

\section{The HS group had a high concentration of serum creatinine and blood urea nitrogen at 21} weeks of age

There was no significant difference in the concentration of serum creatinine or BUN between the 
166 LS group and HS group at 11 weeks of age (Supplementary Figure 1B). However, the HS group

167 showed a significantly higher concentration of both creatinine and BUN compared to the LS

168 group at 21 weeks of age (Supplementary Figure 1C).

169

170

Fibrosis in the kidney and gene expression related to the glomerulus, renal tubules, and

171

172

173

174

175

176

177

178

179

180

181 (Figure 1A).

182

183 The gene expression of kidney injury molecule-1 (Kiml) indicates the degree of interstitial 
184 fibrosis, renal tubular damage, and inflammation (van Timmeren et al. 2006). Neutrophil

185 gelatinase-associated lipocalin (NGAL) is also associated with renal tubular damage in ischemic

186 or inflammatory responses (Mori et al. 2005). In the present study, the expression of Kim 1 and

187 Ngal was significantly higher in the HS group than the LS group at both 11 and 21 weeks of age

188 (Figure 1B). In addition, in the HS group, the expression of these genes was significantly

189 increased at 21 weeks of age compared to 11 weeks of age (Figure 1B).

The expression of the osteopontin gene increases in rat renal fibroblasts with aldosterone (Irita et type 1 collagen (collagen 1$)$ and $\alpha$-smooth muscle actin genes ( $\alpha$ SMA) increases in renal fibrosis

194 (Lekgabe et al. 2005; Wang et al. 2008). In the present study, expression of these genes was significantly higher in the HS group than the LS group at both 11 and 21 weeks of age (Figure 1C). The expression of these genes increased significantly at 21 weeks of age compared to 11 weeks in the HS group only (Figure 1C).

Gene expression related to mitochondrial function, glycolysis, and free fatty acid metabolism in the kidneys

We examined the expression level of some genes related to mitochondrial function, glycolysis, 
202 and fatty acid metabolism at 11 and 21 weeks of age. Gene expression related to glycolysis was

203 measured, and the expression of hexokinase (Hk) 1 and $H k 2$, which catalyze the transfer from

204 glucose to glucose-6-phosphate (G6P), significantly increased in the HS group compared to the

205 LS group at 21 weeks of age. Conversely, the expression of Tp53-induced glycolysis and

206 apoptosis regulator (TIGAR), which alters the concentration of fructose 2,6-bisphosphate

207 (Bensaad et al. 2006), was significantly decreased in the HS group compared to the LS group at

20821 weeks of age (Figure 2 and Supplementary Table 2).

209

210

Peroxisome proliferator-activated receptor $\gamma$ coactivator $1-\alpha(\mathrm{PGC} 1-\alpha)$ is a master regulator of

211 mitochondrial biogenesis, though there was no change between the LS and HS group (Figure 2

212 and 3, and Supplementary Table 2). Proliferator activated receptor $\alpha$ (PPAR $\alpha)$, nuclear

213 respiratory factor 1 (NRF1), and NRF2 are related to mitochondrial biogenesis. Ppara

214 expression was significantly decreased and $\mathrm{Nrfl}$ expression was significantly increased in the HS

215 group compared to the LS group at 21 weeks of age. Otherwise, compared to that in the LS

216 group, $\operatorname{Nrf} 2$ expression was significantly increased at 11 weeks of age and decreased at 21 weeks

217 of age in the HS group (Figure 2 and 3, and Supplementary Table 2).

219 Subsequently, we measured the expression of genes related to oxidative phosphorylation. 
220 Though uncoupling protein 3 (UCP3) is known for uncoupling ATP synthesis from oxidative

221 metabolism, its transcription is regulated by PPAR $\alpha$ (Young et al. 2001). In this study, like

222 Ppara expression, Ucp3 expression decreased significantly in the HS group compared to the LS

223 group at 21 weeks of age. The expression levels of several genes related to the respiratory chain

224 also changed in the HS group; NADH dehydrogenase $4(N d 4)$, alpha-subcomplex $9(\alpha S 9)$,

225 succinate dehydrogenase $\mathrm{b}(S d h b)$ and cytochrome-c $(C y t-c)$ expression significantly decreased

226 in the HS group from that in the LS group at 21 weeks of age. Conversely, in the HS group,

227 expression of cytochrome c oxidase 1 ( $\operatorname{Cox} 1)$ decreased at 11 weeks of age and increased at 21

228 weeks of age, and Cox4 increased significantly at 21 weeks of age (Figure 2 and 3, and

229 Supplementary Table 2).

230

231 Medium-chain acyl-CoA dehydrogenase (MCAD) is an enzyme related to fatty acid oxidation,

232 and its expression decreased significantly in the HS group compared to the LS group at 21 weeks

233 of age. ATP-binding cassette sub-family A member 1 (ABCA1) has a critical role in cellular

234 cholesterol and phospholipid efflux, and its gene expression was significantly increased in the

235 HS group compared to the LS group at 21 weeks of age (Figure 2 and Supplementary Table 2). 
238 To examine energy metabolism in renal damage, we performed a comprehensive metabolomic

239 analysis. The results of the quantification showed metabolites related to glycolysis, including the

240 TCA cycle, and amino acids and their derivatives (Figure 4 and Supplementaly Table 3). By

241 two-way ANOVA analysis, we assessed the significance ( $p$ value) for time (11 weeks and 21

242 weeks) and treatment effects (LS diet and HS diet). The metabolites that were not significantly

243 different ( $\mathrm{p} \geq 0.05)$ regarding time or interaction effects are only represented by the bar plots of

244 21-week-old rats. Other metabolites are represented by bar plots of 11-week-old and 21-week-

245 old rats (Figure 4). The metabolites of kidney tissue significantly changed in the HS group from

246 that in the LS group. Especially, the increased urea and creatinine levels at 21 weeks of age

247 validated that the metabolome analysis was performed successfully.

The levels of 2,3-diphosphoglycerate (2,3-DPG) and phosphoenolpyruvate (PEP), intermediate metabolites of glycolysis, decreased in the HS group at 21 weeks of age. The levels of several amino acids and derivatives such as serine, betaine, alanine, histidine, citruline, proline, arginine and putrescine, increased in the HS group at 21 weeks of age. On the contrary, taurine, $\beta$-alanine, and glutamate were decreased in the HS group at 21 weeks of age. Levels of several metabolites in the TCA cycle also changed; the levels of citrate, cis-acotinate and isocitrate increased, and the levels of succinate and acetyl co-A decreased (Figure 4 and Supplementary Table 3). 
257 Subsequently, we measured the gene expression of enzymes that are involved in the TCA cycle

258 (Figure 5): aconitase is encoded by Aco2, which catalyzes the reversible isomerization of citrate and isocitrate; 2-oxoglutarate dehydrogenase $(\mathrm{OGDH})$ is an enzyme that catalyzes the oxidation of 2-oxoglutarate; succinyl-CoA synthetase (SCS) is encoded by the gene Suclg1, and is an enzyme that hydrolyzes succinyl-CoA into succinate and CoA-SH; fumarate hydratase is encoded by the $F h$ gene and is the enzyme that catalyzes the hydration of fumarate to malate.

The gene expression of $A c o 2, O g d h$, Suclgl, and Fh was significantly decreased in the HS group compared to the LS group at 21 weeks of age (Figure 5).

Furthermore, the amounts of nicotinamide adenine dinucleotide (NAD+) and NADH were measured on metabolome analysis. NAD + plays a central role in cellular energy metabolism and energy production. The NAD+/NADH ratio is often considered as the key of mitochondrial function. Cytoplasmic NAD/NADH ratios range between 60 and 700, and mitochondrial

NAD/NADH ratios are maintained at 7 to 8 (Stein \& Imai 2012). The reduction of

$271 \mathrm{NAD}+/ \mathrm{NADH}$ ratio has been reported in renal injuries in a type 1 diabetic mouse model (Zhu et al. 2014). In the present study, both NAD+ and NADH levels significantly decreased at 21 weeks 
274 (Supplementary Figure 3B). It is well known that mitochondrial Complex I catalyzes the first

275 step of NADH oxidation, and increases the NAD+/NADH ratio. Therefore, we measured the

276 protein expression of NADH dehydrogenase ubiquinone $1 \alpha$ subcomplex subunit 9 (NDUFA9) a

277 subunits of mitochondrial Complex I, at 21 weeks of age, because the level of mRNA expression

278 of NDUFA9 $(\alpha S 9)$ was significantly decreased in the HS group (Figure 2 and 3). However, there

279 was no significant change between the LS group and HS group (Supplementary Figure 3B).

\section{Discussion}

Consistent with previous reports (Hisaki et al. 2005; Karlsen et al. 1997; Kato et al. 2010; Rapp

\& Dene 1985), DS rats fed an HS diet showed high blood pressure, an increased concentration of serum creatinine and BUN, and an increased fibrotic zone in their kidneys at 21 weeks of age.

We demonstrated that the metabolites levels in the first half of the TCA cycle increased; the level of succinate in the latter half of the TCA cycle decreased in DS rats in CKD with CRS, and the gene expression related to energy metabolism also significantly changed.

The DS rats fed a high-salt diet showed an increase in urinary protein expression and serum creatinine levels, and a decrease in creatinine clearance. They also exhibited injured glomeruli characterized by sclerosis enlarged glomerular size, and severe tubulointerstitial fibrosis on 
292 histochemical analysis (Du et al. 2009). Moreover, DS rats treated with vasopressin type 2

293 receptor antagonist showed an improvement in cardiac dysfunction and kidney dysfunction

294 simultaneously (Ishikawa et al. 2013; Morooka et al. 2012). Treatment with the calcium

295 sensitizer and the AT1-receptor antagonist in DS rats also ameliorate cardiac dysfunction and

296 kidney injury simultaneously (Biala et al. 2011). We showed a close correlation among systolic

297 blood pressure, cardiac dysfunction, and renal damage. These findings suggested that the DS rat

298 is a useful model of CKD with CRS. Although this model did not fully recapitulate human

299 hypertensive renal disease, the Dahl rat model nevertheless may have many advantages in

300 preclinical studies for determination of suitable candidates for biomarker and drug discovery.

In the present study, the metabolic profiles in kidney tissues revealed increased levels of citrate and isocitrate, decreased gene expression of $O g d h$ and $S c s$, which are enzymes that metabolize citrate and isocitrate, respectively, and decreased levels of succinate and acetyl co-A, components of the TCA cycle. This profile consisting of mostly decreased gene expression related to mitochondrial function, indicated mitochondrial dysfunction in both hypertension and

long-lasting hypertension. Zheleznova et al. reported oxygen deficiencies in DS rats using mitochondrial proteomic analyses; mitochondrial protein expression was depressed with decreased oxygen utilization in the renal medullary thick ascending limb of the Dahl rats 
310 (Zheleznova et al. 2012). Renal mitochondrial damage has also been reported in various models

311 of hypertension, such as the spontaneous hypertension rat, 2-kidney/1-clip model rat,

312 ischemia/reperfusion injury, and renal hypertension in pigs (Eirin et al. 2015). Therefore,

313 mitochondrial damage may be the hallmark of CKD due to hypertension. In patients with kidney

314 disease, urine metabolomics have revealed that metabolites linked to mitochondria are

315 consistently decreased in the human diabetic kidney (Sharma et al. 2013). Others have also

316 reported the downregulation of genes related to oxidative metabolism in patients with CKD on

317 peritoneal dialysis (Zaza et al. 2013).

318

319 The mechanism underling the renal mitochondrial damage in Dahl rat was not determined in the

320 present study. Hypertension is commonly associated with mechanical stretch, increased

321 production of ROS, and extracellular matrix turnover and fibrosis, which in turn alter the

322 structure and function of cellular organelles including mitochondria. In Dahl rats, enhanced ROS

323 production has been detected in various organs of salt hypertensive Dahl rats including the

324 kidney (Trolliet et al. 2001; Vokurkova et al. 2015), heart (Kato et al. 2010), liver (Kato et al.

325 2012), and blood vessels (Swei et al. 1997; Trolliet et al. 2001). In addition, serum

326 concentrations of pro-inflammatory cytokines are also elevated (Kato et al. 2012). These factors

327 may contribute to the development of mitochondrial dysfunction, which in turn causes podocyte 
328 injury, tubular epithelial cell damage, and endothelial dysfunction (Che et al. 2014).

329 Mitochondrial dysfunction also stimulates TGF- $\beta$ signaling and fibrosis through mitochondrial-

330 derived ROS (Che et al. 2014; Jain et al. 2013).

332 There are two clinical implications of the present study. First, its use for biomarker development

333 should be mentioned. Metabolites changed as the disease progressed. Therefore, metabolites in

334 blood and urine samples could be candidates for a biomarker for the early detection of CKD or

335 the prediction of worsening disease (Cisek et al. 2015; Sharma et al. 2013; Zaza et al. 2013). The

336 second implication is the development of therapeutic targets. Though it remains unclear how

337 renal dysfunction cause abnormal metabolic status in mitochondria, the mitochondria could also

338 be a target of therapy for hypertensive kidney disease (Che et al. 2014; Ishimoto \& Inagi 2015).

339 For example, activation of PGC1 $\alpha$ using a transgenic model prevented acute kidney injury (Tran

340 et al. 2011). In addition, quinone analogues such as MitoQ are mitochondrial ROS modulators

341 and other antioxidants such as omega-3 polyunsaturated fatty acids, $\mathrm{N}$-acetylcysteine, and

342 allopurinol are all candidates for mitochondrial ROS modulators (Ishimoto \& Inagi 2015). The

343 SIRT 1 activator resveratrol improves mitochondrial function and reduces oxidative stress

344 (Wang et al. 2015); clinical trials have been planned to investigate usage of these modulators in

345 patients with CKD. The present study as well as previous reports has highlighted possible 
346 metabolic therapies for CKD.

348 This study aimed to characterize one CKD model and the imposed study limitations should be stated. Data on other CKD models were not examined in this study. Metabolome analyses are a snapshot of changes; hence, the flow of metabolites was an estimation. Microarray analysis was not performed, and gene expression was measured by quantitative reverse transcriptionpolymerase chain reaction (RT-PCR). Therefore, pathway analysis, such as KEGG, could not be performed in the present study. We analyzed whole kidney including tubules and glomeruli; so we could not isolate the part the kidney undergoing the metabolic change. In addition, this study was observational and we did not intervene in this model. Therefore, another set of experiments is needed to clarify whether the metabolic changes is the cause or the consequence of kidney injury. However, this model is still useful for preclinical studies since there is an increasing recognition that the kidney plays an important role in complex inter-organ communication, which affects the development of cardiovascular diseases.

\section{Conclusions}

362 DS rats fed an HS diet are deemed a suitable model of CKD. Gene expression and metabolites related to energy metabolism and mitochondria in the kidney significantly changed in DS rats 
364 with hypertension in accordance with the progression of renal injury. 
365

366

367

368

369

370

371

372

373

374

375

376

377

378

379

380

381

382

383

384

385

386

387

388

389

390

391

392

393

394

395

396

397

398

\section{References}

Bensaad K, Tsuruta A, Selak MA, Vidal MN, Nakano K, Bartrons R, Gottlieb E, and Vousden KH. 2006. TIGAR, a p53-inducible regulator of glycolysis and apoptosis. Cell 126:107-120.

Biala A, Finckenberg P, Korpi A, Loytainen M, Martonen E, Levijoki J, and Mervaala E. 2011. Cardiovascular effects of the combination of levosimendan and valsartan in hypertensive Dahl/Rapp rats. J Physiol Pharmacol 62:275-285.

Bidani AK, and Griffin KA. 2004. Pathophysiology of hypertensive renal damage: implications for therapy. Hypertension 44:595-601.

Blasi ER, Rocha R, Rudolph AE, Blomme EA, Polly ML, and McMahon EG. 2003. Aldosterone/salt induces renal inflammation and fibrosis in hypertensive rats. Kidney Int 63:1791-1800.

Boute N, Gribouval O, Roselli S, Benessy F, Lee H, Fuchshuber A, Dahan K, Gubler MC, Niaudet P, and Antignac C. 2000. NPHS2, encoding the glomerular protein podocin, is mutated in autosomal recessive steroid-resistant nephrotic syndrome. Nat Genet 24:349-354.

Che R, Yuan Y, Huang S, and Zhang A. 2014. Mitochondrial dysfunction in the pathophysiology of renal diseases. Am J Physiol Renal Physiol 306:F367-378.

Cisek K, Krochmal M, Klein J, and Mischak H. 2015. The application of multi-omics and systems biology to identify therapeutic targets in chronic kidney disease. Nephrol Dial Transplant.

Du J, Fan YY, Hitomi H, Kiyomoto H, Kimura S, Kong CZ, Noma T, Kohno M, Nishiyama A, and Nakano D. 2009. Mineralocorticoid receptor blockade and calcium channel blockade have different renoprotective effects on glomerular and interstitial injury in rats. Am J Physiol Renal Physiol 297:F802-808.

Eirin A, Lerman A, and Lerman LO. 2015. Mitochondria: a pathogenic paradigm in hypertensive renal disease. Hypertension 65:264-270.

Eirin A, and Lerman LO. 2013. Darkness at the end of the tunnel: poststenotic kidney injury. Physiology (Bethesda) 28:245-253.

Hillege HL, Girbes AR, de Kam PJ, Boomsma F, de Zeeuw D, Charlesworth A, Hampton JR, and van Veldhuisen DJ. 2000. Renal function, neurohormonal activation, and survival in patients with chronic heart failure. Circulation 102:203-210.

Hisaki R, Fujita H, Saito F, and Kushiro T. 2005. Tempol attenuates the development of hypertensive renal injury in Dahl salt-sensitive rats. Am J Hypertens 18:707-713.

Inoko M, Kihara Y, Morii I, Fujiwara H, and Sasayama S. 1994. Transition from compensatory hypertrophy to dilated, failing left ventricles in Dahl salt-sensitive rats. Am J Physiol 267:H24712482. 
399

400

401

402

403

404

405

406

407

408

409

410

411

412

413

414

415

416

417

418

419

420

421

422

423

424

425

426

427

428

429

430

431

432

433

434

Inuzuka Y, Okuda J, Kawashima T, Kato T, Niizuma S, Tamaki Y, Iwanaga Y, Yoshida Y, Kosugi R, Watanabe-Maeda K, Machida Y, Tsuji S, Aburatani H, Izumi T, Kita T, and Shioi T. 2009. Suppression of phosphoinositide 3-kinase prevents cardiac aging in mice. Circulation 120:16951703.

Irita J, Okura T, Kurata M, Miyoshi K, Fukuoka T, and Higaki J. 2008. Osteopontin in rat renal fibroblasts: functional properties and transcriptional regulation by aldosterone. Hypertension 51:507-513.

Ishikawa M, Kobayashi N, Sugiyama F, Onoda S, and Ishimitsu T. 2013. Renoprotective effect of vasopressin v2 receptor antagonist tolvaptan in Dahl rats with end-stage heart failure. Int Heart $J$ 54:98-106.

Ishimoto Y, and Inagi R. 2015. Mitochondria: a therapeutic target in acute kidney injury. Nephrol Dial Transplant.

Jain M, Rivera S, Monclus EA, Synenki L, Zirk A, Eisenbart J, Feghali-Bostwick C, Mutlu GM, Budinger GR, and Chandel NS. 2013. Mitochondrial reactive oxygen species regulate transforming growth factor-beta signaling. J Biol Chem 288:770-777.

Karlsen FM, Andersen CB, Leyssac PP, and Holstein-Rathlou NH. 1997. Dynamic autoregulation and renal injury in Dahl rats. Hypertension 30:975-983.

Kato T, Niizuma S, Inuzuka Y, Kawashima T, Okuda J, Kawamoto A, Tamaki Y, Iwanaga Y, Soga T, Kita T, Kimura T, and Shioi T. 2012. Analysis of liver metabolism in a rat model of heart failure. Int J Cardiol 161:130-136.

Kato T, Niizuma S, Inuzuka Y, Kawashima T, Okuda J, Tamaki Y, Iwanaga Y, Narazaki M, Matsuda T, Soga T, Kita T, Kimura T, and Shioi T. 2010. Analysis of metabolic remodeling in compensated left ventricular hypertrophy and heart failure. Circ Heart Fail 3:420-430.

Kawamoto A, Kato T, Shioi T, Okuda J, Kawashima T, Tamaki Y, Niizuma S, Tanada Y, Takemura G, Narazaki M, Matsuda T, and Kimura T. 2015. Measurement of technetium-99m sestamibi signals in rats administered a mitochondrial uncoupler and in a rat model of heart failure. PLoS One 10:e0117091.

Lekgabe ED, Kiriazis H, Zhao C, Xu Q, Moore XL, Su Y, Bathgate RA, Du XJ, and Samuel CS. 2005. Relaxin reverses cardiac and renal fibrosis in spontaneously hypertensive rats. Hypertension 46:412-418.

Lozano R, Naghavi M, Foreman K, Lim S, Shibuya K, Aboyans V, Abraham J, Adair T, Aggarwal R, Ahn SY, Alvarado M, Anderson HR, Anderson LM, Andrews KG, Atkinson C, Baddour LM, Barker-Collo S, Bartels DH, Bell ML, Benjamin EJ, Bennett D, Bhalla K, Bikbov B, Bin Abdulhak A, Birbeck G, Blyth F, Bolliger I, Boufous S, Bucello C, Burch M, Burney P, Carapetis J, Chen H, Chou D, Chugh SS, Coffeng LE, Colan SD, Colquhoun S, Colson KE, Condon J, Connor MD, Cooper LT, Corriere M, Cortinovis M, de Vaccaro KC, Couser W, Cowie 
435

436

437

438

439

440

441

442

443

444

445

446

447

448

449

450

451

452

453

454

455

456

457

458

459

460

461

462

463

464

465

466

467

468

469

470

BC, Criqui MH, Cross M, Dabhadkar KC, Dahodwala N, De Leo D, Degenhardt L, Delossantos A, Denenberg J, Des Jarlais DC, Dharmaratne SD, Dorsey ER, Driscoll T, Duber H, Ebel B, Erwin PJ, Espindola P, Ezzati M, Feigin V, Flaxman AD, Forouzanfar MH, Fowkes FG, Franklin R, Fransen M, Freeman MK, Gabriel SE, Gakidou E, Gaspari F, Gillum RF, Gonzalez-Medina D, Halasa YA, Haring D, Harrison JE, Havmoeller R, Hay RJ, Hoen B, Hotez PJ, Hoy D, Jacobsen KH, James SL, Jasrasaria R, Jayaraman S, Johns N, Karthikeyan G, Kassebaum N, Keren A, Khoo JP, Knowlton LM, Kobusingye O, Koranteng A, Krishnamurthi R, Lipnick M, Lipshultz SE, Ohno SL, Mabweijano J, MacIntyre MF, Mallinger L, March L, Marks GB, Marks R, Matsumori A, Matzopoulos R, Mayosi BM, McAnulty JH, McDermott MM, McGrath J, Mensah GA, Merriman TR, Michaud C, Miller M, Miller TR, Mock C, Mocumbi AO, Mokdad AA, Moran A, Mulholland K, Nair MN, Naldi L, Narayan KM, Nasseri K, Norman P, O'Donnell M, Omer SB, Ortblad K, Osborne R, Ozgediz D, Pahari B, Pandian JD, Rivero AP, Padilla RP, Perez-Ruiz F, Perico N, Phillips D, Pierce K, Pope CA, 3rd, Porrini E, Pourmalek F, Raju M, Ranganathan D, Rehm JT, Rein DB, Remuzzi G, Rivara FP, Roberts T, De Leon FR, Rosenfeld LC, Rushton L, Sacco RL, Salomon JA, Sampson U, Sanman E, Schwebel DC, Segui-Gomez M, Shepard DS, Singh D, Singleton J, Sliwa K, Smith E, Steer A, Taylor JA, Thomas B, Tleyjeh IM, Towbin JA, Truelsen T, Undurraga EA, Venketasubramanian N, Vijayakumar L, Vos T, Wagner GR, Wang M, Wang W, Watt K, Weinstock MA, Weintraub R, Wilkinson JD, Woolf AD, Wulf S, Yeh PH, Yip P, Zabetian A, Zheng ZJ, Lopez AD, Murray CJ, AlMazroa MA, and Memish ZA. 2012. Global and regional mortality from 235 causes of death for 20 age groups in 1990 and 2010: a systematic analysis for the Global Burden of Disease Study 2010. Lancet 380:2095-2128.

Mori K, Lee HT, Rapoport D, Drexler IR, Foster K, Yang J, Schmidt-Ott KM, Chen X, Li JY, Weiss S, Mishra J, Cheema FH, Markowitz G, Suganami T, Sawai K, Mukoyama M, Kunis C, D'Agati V, Devarajan P, and Barasch J. 2005. Endocytic delivery of lipocalin-siderophore-iron complex rescues the kidney from ischemia-reperfusion injury. J Clin Invest 115:610-621.

Morooka H, Iwanaga Y, Tamaki Y, Takase T, Akahoshi Y, Nakano Y, Fujiki H, and Miyazaki S. 2012. Chronic administration of oral vasopressin type 2 receptor antagonist tolvaptan exerts both myocardial and renal protective effects in rats with hypertensive heart failure. Circ Heart Fail 5:484-492.

Niizuma S, Inuzuka Y, Okuda J, Kato T, Kawashima T, Tamaki Y, Iwanaga Y, Yoshida Y, Kosugi R, Watanabe-Maeda K, Machida Y, Tsuji S, Aburatani H, Izumi T, Kita T, Kimura T, and Shioi T. 2012. Effect of persistent activation of phosphoinositide 3-kinase on heart. Life Sci 90:619-628.

Nishiyama A, Yoshizumi M, Hitomi H, Kagami S, Kondo S, Miyatake A, Fukunaga M, Tamaki T, Kiyomoto H, Kohno M, Shokoji T, Kimura S, and Abe Y. 2004. The SOD mimetic tempol ameliorates glomerular injury and reduces mitogen-activated protein kinase activity in Dahl saltsensitive rats. J Am Soc Nephrol 15:306-315.

Peer] reviewing PDF | (2016:11:14512:1:1:NEW 4 Apr 2017) 
471 Nourbakhsh N, and Singh P. 2014. Role of renal oxygenation and mitochondrial function in the

472

473

474

475

476

477

478

479

480

481

482

483

484

485

486

487

488

489

490

491

492

493

494

495

496

497

498

499

500

501

502

503

504 pathophysiology of acute kidney injury. Nephron Clin Pract 127:149-152.

Okuda J, Niizuma S, Shioi T, Kato T, Inuzuka Y, Kawashima T, Tamaki Y, Kawamoto A, Tanada Y, Iwanaga Y, Narazaki M, Matsuda T, Adachi S, Soga T, Takemura G, Kondoh H, Kita T, and Kimura T. 2013. Persistent overexpression of phosphoglycerate mutase, a glycolytic enzyme, modifies energy metabolism and reduces stress resistance of heart in mice. PLoS One 8:e72173.

Rapp JP, and Dene H. 1985. Development and characteristics of inbred strains of Dahl salt-sensitive and salt-resistant rats. Hypertension 7:340-349.

Rubattu S, Mennuni S, Testa M, Mennuni M, Pierelli G, Pagliaro B, Gabriele E, Coluccia R, Autore C, and Volpe M. 2013. Pathogenesis of chronic cardiorenal syndrome: is there a role for oxidative stress? Int J Mol Sci 14:23011-23032.

Ruotsalainen V, Ljungberg P, Wartiovaara J, Lenkkeri U, Kestila M, Jalanko H, Holmberg C, and Tryggvason K. 1999. Nephrin is specifically located at the slit diaphragm of glomerular podocytes. Proc Natl Acad Sci U S A 96:7962-7967.

Sharma K, Karl B, Mathew AV, Gangoiti JA, Wassel CL, Saito R, Pu M, Sharma S, You YH, Wang L, Diamond-Stanic M, Lindenmeyer MT, Forsblom C, Wu W, Ix JH, Ideker T, Kopp JB, Nigam SK, Cohen CD, Groop PH, Barshop BA, Natarajan L, Nyhan WL, and Naviaux RK. 2013. Metabolomics reveals signature of mitochondrial dysfunction in diabetic kidney disease. $\mathrm{J} \mathrm{Am}$ Soc Nephrol 24:1901-1912.

Smith GL, Lichtman JH, Bracken MB, Shlipak MG, Phillips CO, DiCapua P, and Krumholz HM. 2006. Renal impairment and outcomes in heart failure: systematic review and meta-analysis. $J$ Am Coll Cardiol 47:1987-1996.

Stein LR, and Imai S. 2012. The dynamic regulation of NAD metabolism in mitochondria. Trends Endocrinol Metab 23:420-428.

Swei A, Lacy F, DeLano FA, and Schmid-Schonbein GW. 1997. Oxidative stress in the Dahl hypertensive rat. Hypertension 30:1628-1633.

Tanada Y, Shioi T, Kato T, Kawamoto A, Okuda J, and Kimura T. 2015. Branched-chain amino acids ameliorate heart failure with cardiac cachexia in rats. Life Sci 137:20-27.

Tran M, Tam D, Bardia A, Bhasin M, Rowe GC, Kher A, Zsengeller ZK, Akhavan-Sharif MR, Khankin EV, Saintgeniez M, David S, Burstein D, Karumanchi SA, Stillman IE, Arany Z, and Parikh SM. 2011. PGC-1alpha promotes recovery after acute kidney injury during systemic inflammation in mice. J Clin Invest 121:4003-4014.

Trolliet MR, Rudd MA, and Loscalzo J. 2001. Oxidative stress and renal dysfunction in salt-sensitive hypertension. Kidney Blood Press Res 24:116-123. 
505

506

507

508

509

510

511

512

513

514

515

516

517

518

519

520

521

522

523

524

525

526

527

528 van Timmeren MM, Bakker SJ, Vaidya VS, Bailly V, Schuurs TA, Damman J, Stegeman CA, Bonventre JV, and van Goor H. 2006. Tubular kidney injury molecule-1 in protein-overload nephropathy. Am J Physiol Renal Physiol 291:F456-464.

Vokurkova M, Rauchova H, Rezacova L, Vaneckova I, and Zicha J. 2015. ROS production is increased in the kidney but not in the brain of Dahl rats with salt hypertension elicited in adulthood. Physiol Res 64:303-312.

Wang H, Guan Y, Karamercan MA, Ye L, Bhatti T, Becker LB, Baur JA, and Sims CA. 2015. Resveratrol Rescues Kidney Mitochondrial Function Following Hemorrhagic Shock. Shock 44:173-180.

Wang L, Lee JY, Kwak JH, He Y, Kim SI, and Choi ME. 2008. Protective effects of low-dose carbon monoxide against renal fibrosis induced by unilateral ureteral obstruction. Am J Physiol Renal Physiol 294:F508-517.

Young ME, Patil S, Ying J, Depre C, Ahuja HS, Shipley GL, Stepkowski SM, Davies PJ, and Taegtmeyer H. 2001. Uncoupling protein 3 transcription is regulated by peroxisome proliferator-activated receptor (alpha) in the adult rodent heart. FASEB $J$ 15:833-845.

Zaza G, Granata S, Masola V, Rugiu C, Fantin F, Gesualdo L, Schena FP, and Lupo A. 2013. Downregulation of nuclear-encoded genes of oxidative metabolism in dialyzed chronic kidney disease patients. PLoS One 8:e77847.

Zheleznova NN, Yang C, Ryan RP, Halligan BD, Liang M, Greene AS, and Cowley AW, Jr. 2012. Mitochondrial proteomic analysis reveals deficiencies in oxygen utilization in medullary thick ascending limb of Henle in the Dahl salt-sensitive rat. Physiol Genomics 44:829-842.

Zhu S, Yang Y, Hu J, Qian L, Jiang Y, Li X, Yang Q, Bai H, and Chen Q. 2014. Wld(S) ameliorates renal injury in a type 1 diabetic mouse model. Am J Physiol Renal Physiol 306:F1348-1356. 


\section{Figure 1}

Gene expression related to the glomerulus, renal tube, and fibrosis.(A) NPHS1. (B) NPHS2. (C) KIM1. (D) NGAL. (E) Osteopontin. (F) Collagen 1. (G) $\alpha$ SMA.

At 11 weeks of age, the expressions of Kim1, Ngal, osteopontin, and collagen 1 were significantly increased in the HS group compared to the LS group. At 21 weeks of age, the expression of Nphs1 significantly decreased in the HS group compared to the LS group. Conversely, the expressions of Nphs2, Kim1, Ngal, osteopontin, collagen 1 , and $\alpha$ SMA significantly increased in the HS group compared to the LS group. LS11week, $n=8$; LS-21 week, $n=12$; HS-11 week, $n=8$; HS-21 week, $n=11 . * p<0.05$ versus LS-C group; $* p<0.05$ versus rats at 11 weeks of age.

A

(Fold)

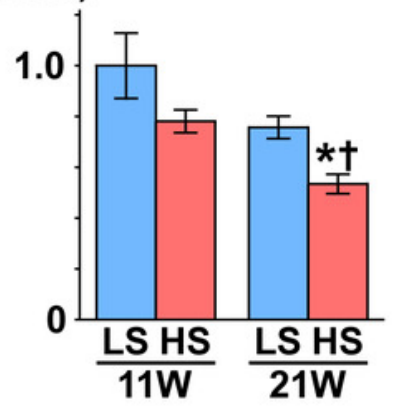

E

(Fold)

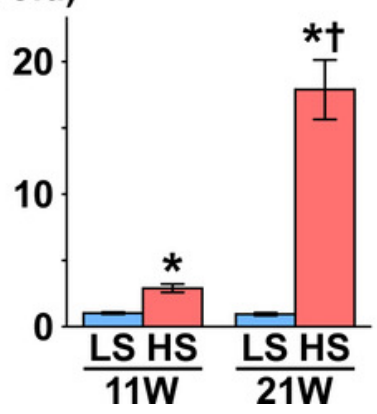

B

(Fold)

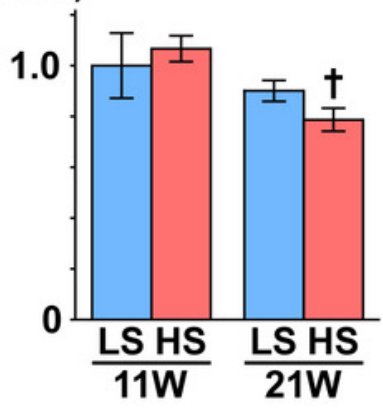

$\mathbf{F}$

(Fold)

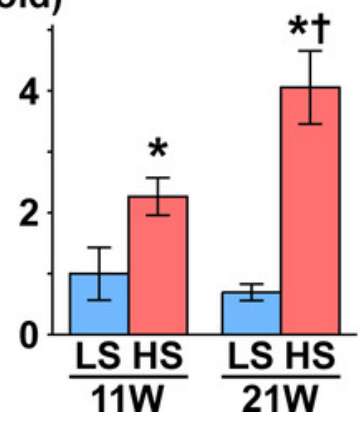

C (Fold)

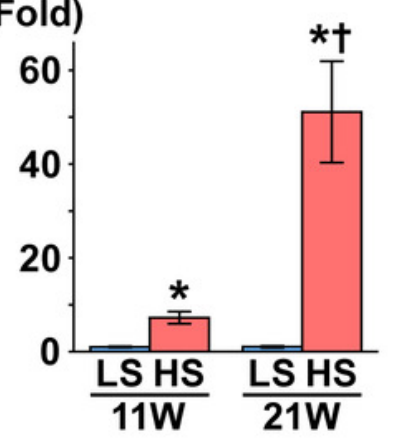

G

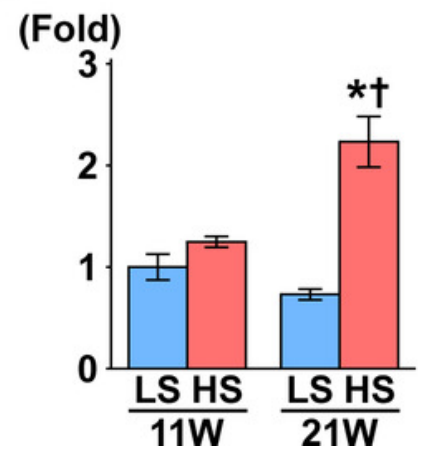

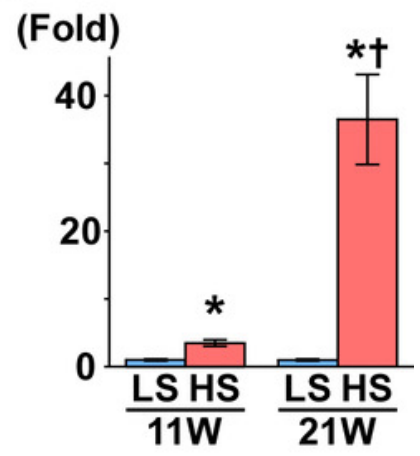


Figure 2

Gene expression related to (A) glycolysis, (B) fatty acids metabolism, and (C)mitochondrial function.

LS-11week, $n=8$; LS-21week, $n=12$; HS-11week, $n=8$; HS-21week, $n=11$. 
A

\begin{tabular}{|l|l|l|}
\hline & 11week & 21week \\
\hline Glut1 & & \\
\hline Glut4 & & \\
\hline HK1 & & \\
\hline HK2 & & \\
\hline PFK & & \\
\hline GAPDH & & \\
\hline PGAM2 & & \\
\hline TIGAR & & \\
\hline HIF-1a & & \\
\hline ACLY & & \\
\hline
\end{tabular}

B

\begin{tabular}{|l|l|l|}
\hline & 11week & 21week \\
\hline ACS & & \\
\hline CPT-1 & & \\
\hline ACC $\alpha$ & & \\
\hline ACC $\beta$ & & \\
\hline FAT/CD36 & & \\
\hline VLCAD & & \\
\hline LCAD & & \\
\hline MCAD & & \\
\hline ABCA1 & & \\
\hline SREBF1 & & \\
\hline SREBF2 & & \\
\hline
\end{tabular}

C

\begin{tabular}{|l|l|l|}
\hline & 11week & 21week \\
\hline PGC1a & & \\
\hline PPARa & & \\
\hline NRF1 & & \\
\hline NRF2 & & \\
\hline TFAM & & \\
\hline UCP3 & & \\
\hline ANT & & \\
\hline SDHB & & \\
\hline aS9 & & \\
\hline ND4 & & \\
\hline Cyt-b & & \\
\hline Cyt-c & & \\
\hline COX1 & & \\
\hline COX4 & & \\
\hline COX5a & & \\
\hline
\end{tabular}

ie:Peg Increased in HS compared to LS $(p<0.05)$ 
Figure 3

Gene expression related to mitochondrial function. (A) PGC1- $\alpha$. (B) PPAR $\alpha$. (C) SDHB.(D) aS9. (E) COX1. (F) COX4.

At 11 weeks of age, the expression of COX4 was significantly decreased in the HS group compared to the LS group. At 21 weeks of age, the expressions of PPAR $\alpha$, SDHB, $\alpha$ S9, COX1, and COX 4 significantly decreased in the HS group compared to the LS group. LS-11week, $n=8$; LS-21week, $n=12$; HS-11week, $n=8$; HS21 week, $n=11 . * p<0.05$ versus LS-C group; $* p<0.05$ versus rats at 11 weeks of age.

A

(Fold)

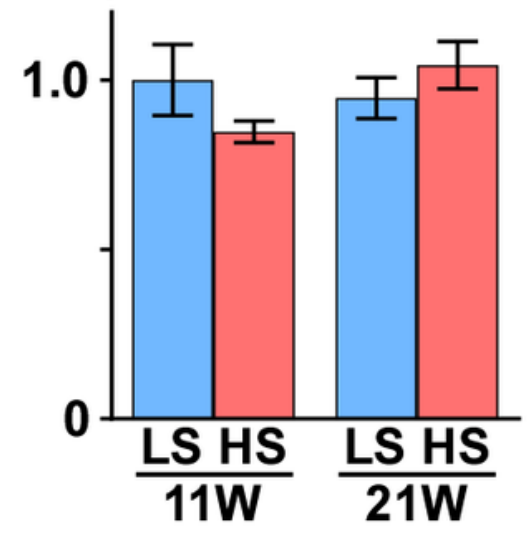

D

(Fold)

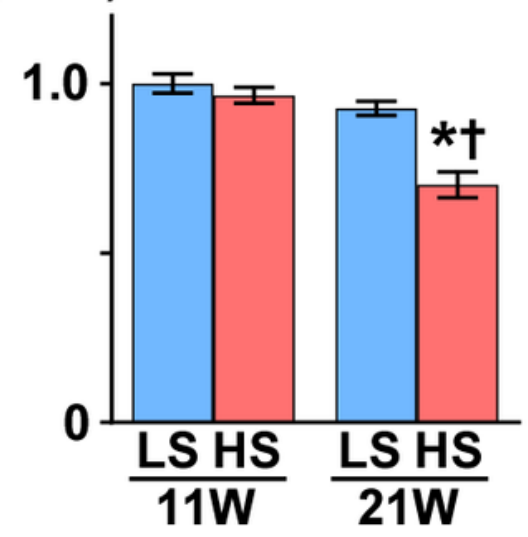

B

(Fold)

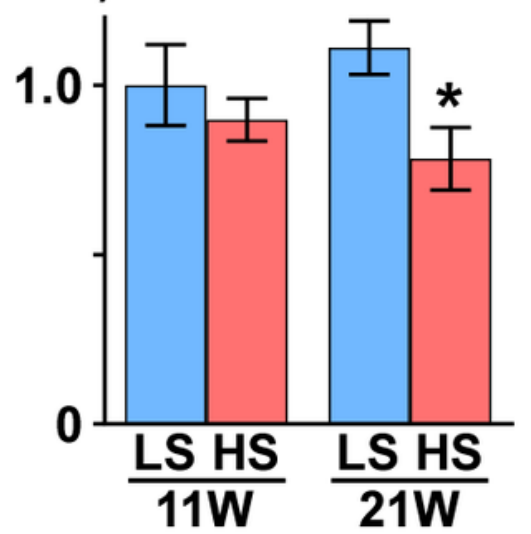

C

(Fold)

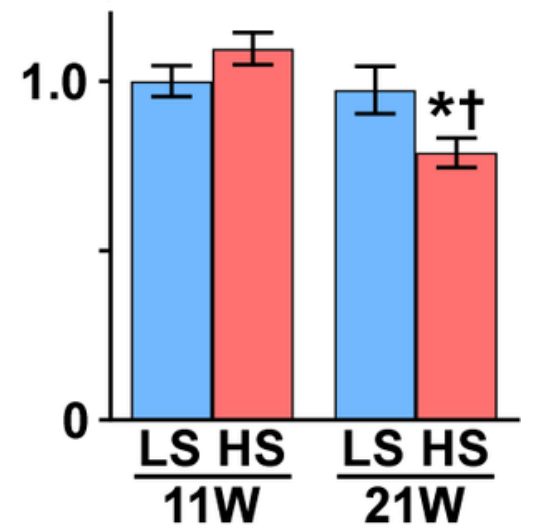

E

(Fold)

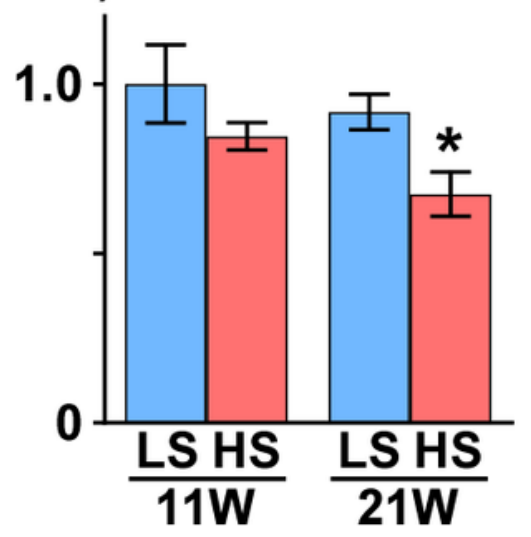

F

(Fold)

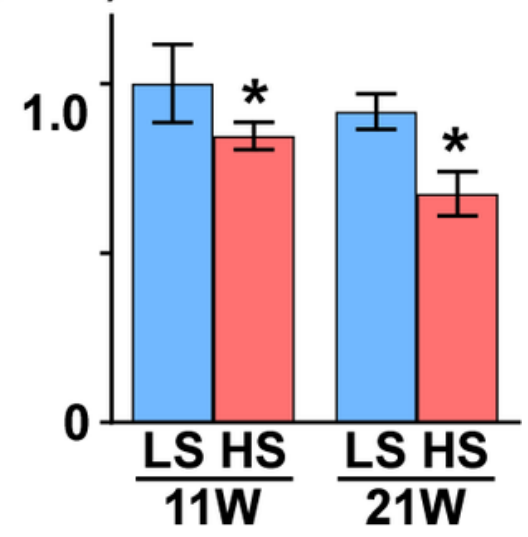




\title{
Figure 4
}

\author{
Metabolites in kidney tissue.
}

After assessing the $p$ value for the time effect (11 weeks and 21 weeks) and the treatment effect (LS diet and HS diet), the metabolites that were not significantly different $(p \geq 0.05)$ regarding time or interaction effect are only represent by bar plots of 21-week-old rats. Other metabolites are represented by bar plots of 11-week-old and 21-weeks-old rats. In the HS group at 21 weeks of age, the levels of 2,3-

diphosphoglycerate (2,3-DPG) and phosphoenolpyruvate (PEP), intermediate metabolites of glycolysis, decreased. The levels of several amino acids and their derivatives also changed in rats in the HS group at 21 weeks of age. The amount of urea, creatinine, and citrulline increased at 21 weeks of age. The levels of several metabolites in the TCA cycle also changed; the levels of citrate and isocitrate increased while that of succinate and acetyl co-A decreased. LS-11week, $n=8$; LS-11week, $n=12$; HS-11week, $n=8$; HS-21week, $\mathrm{n}=11$. 


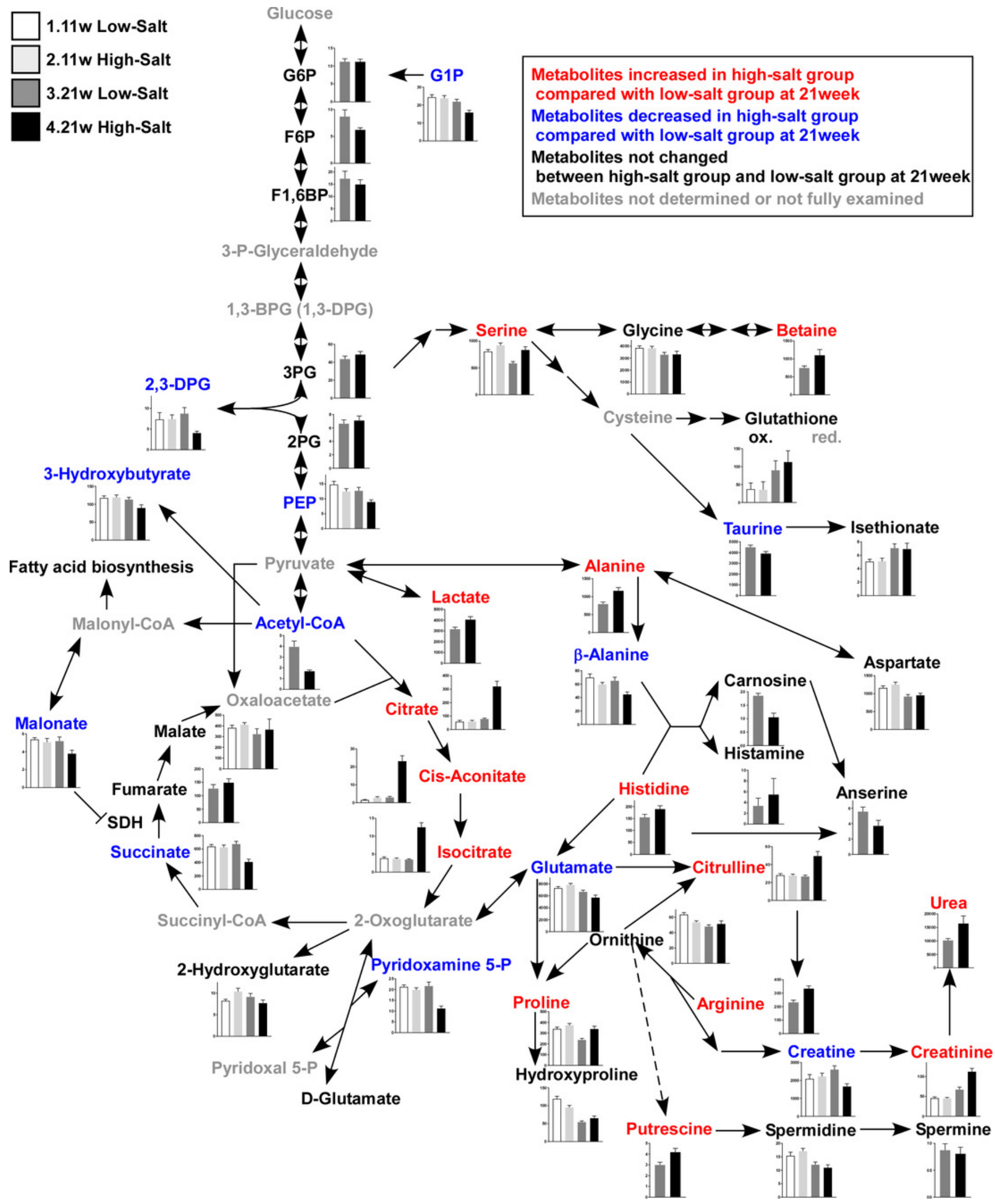


Figure 5

Gene expression of enzymes in the TCA cycle. (A) CS. (B) Aco2. (C) OGDH. (D) Sulg1.(E) Fh. (F) MDH2.

The expressions of Aco2, Ogdh, Suclg1, and Fh significantly decreased in the HS group at 21 weeks of age. These results indicate mitochondrial dysfunction related to the TCA cycle. LS-11week, $n=8$; LS-21week, $n=12$; HS-11 week, $n=8$; HS-21week, $n=11 * p<0.05$ versus LS-C group; $* p<0.05$ versus rats at 11 weeks of age.

A

(Fold)

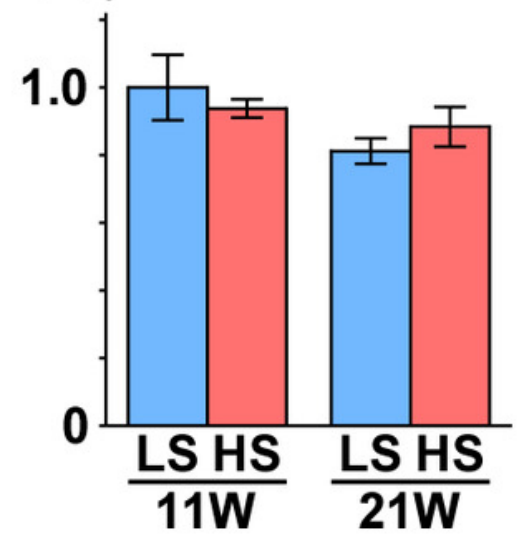

D

(Fold)

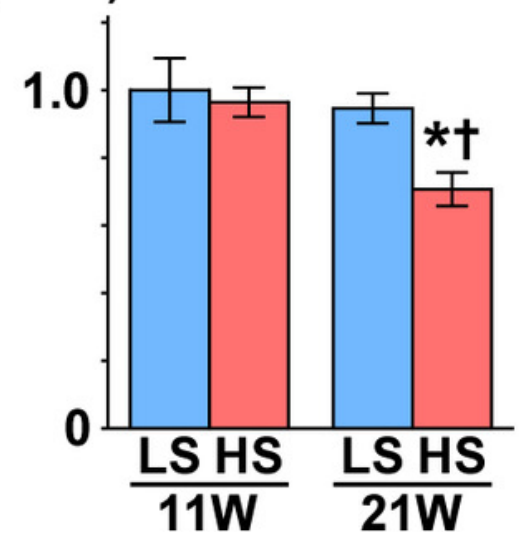

B

(Fold)

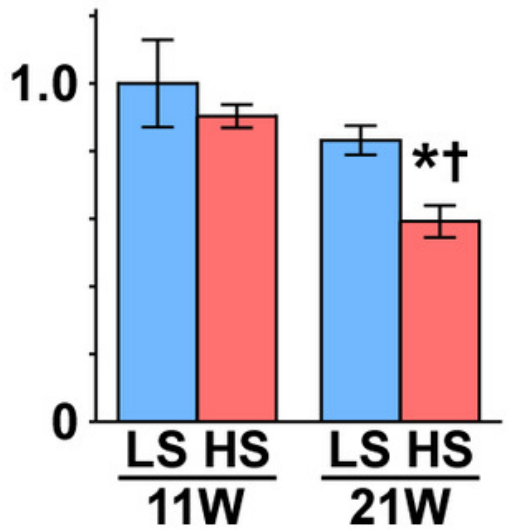

E

(Fold)

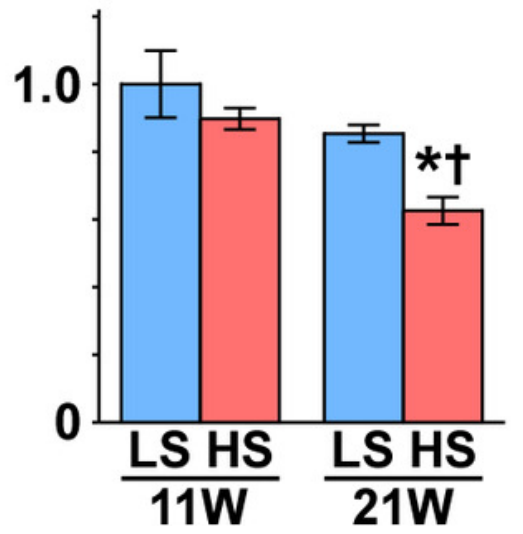

C

(Fold)

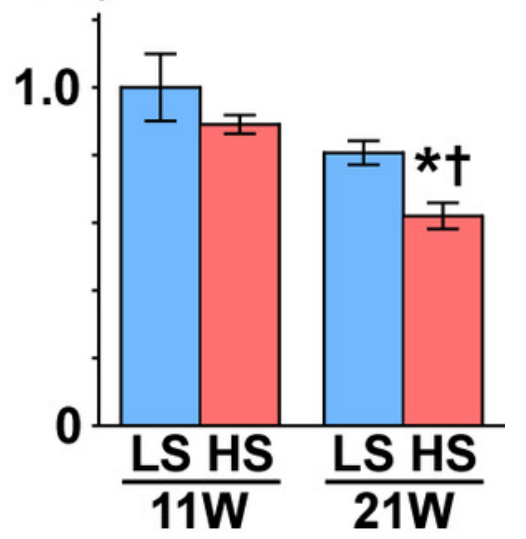

F

(Fold)

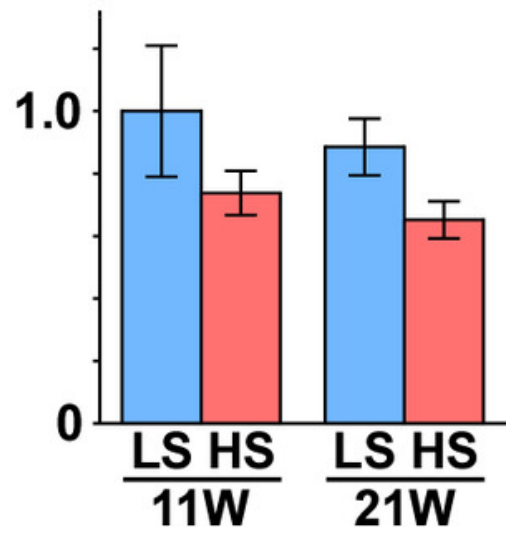

